\title{
Association of Sedentary Behavior with the Expression Levels of Biomarkers in Colorectal Cancer: Clinical Analysis of 228 Patients
}

\author{
Ting Yu, ${ }^{1}$ Qin Lu, ${ }^{1}$ Xilong Ou, ${ }^{1}$ Dazhong $\mathrm{Cao}^{1}$ and Qian Yu ${ }^{1}$ \\ ${ }^{1}$ Department of Gastroenterology, Affiliated Zhongda Hospital of Southeast University, Nanjing, Jiangsu, P.R. \\ China
}

\begin{abstract}
There is an association between sedentary behavior and the risk of colorectal cancer (CRC), but the underlying mechanism is unclear. CRC is characterized by the changes in the expression levels of biomarkers, including voltage-gated proton channel Hv1, matrix-remodeling associated 5 (MXRA5), DEK (inducing positive supercoils into circular DNA) and protein inhibitor of activated signal transducer and activators of transcription 3 (PIAS3). Thus, sedentary behavior may affect the expression levels of these biomarkers in the colorectal tissue. Here, we recruited 228 CRC patients (128 males, $57.8 \pm 7.8$ years; 100 females, $57.7 \pm 7.5$ years) and 80 healthy subjects (48 males, $57.5 \pm 6.8$ years; 32 females, $56.9 \pm 6.5$ years) from March 7th, 2010 to May 6th, 2012. All the subjects were unrelated Han Chinese with the similar cultural and economic background. All the subjects were interviewed concerning sedentary time (sitting time categories: less than 1, 1-3, 4-6, and more than $6 \mathrm{~h} /$ day). The daily sedentary time of most CRC patients was more than $4 \mathrm{~h} /$ day, while the sedentary time of most healthy subjects was less than $3 \mathrm{~h} /$ day. The expression levels of Hv1, MXRA5 and DEK mRNAs and proteins were higher in CRC tissues and the levels of PIAS3 mRNA and protein were lower when the daily sedentary time was longer in CRC patients $(p<0.05)$. The daily sedentary time was correlated with the protein levels of CRC biomarkers. Furthermore, the sedentary time was positively related with body mass index but not daily calorie intake.
\end{abstract}

Keywords: biomarker; body mass index; colorectal cancer; daily calorie intake; daily sedentary time Tohoku J. Exp. Med., 2014 March, 232 (3), 167-176. (C) 2014 Tohoku University Medical Press

\section{Introduction}

Colorectal cancer (CRC) is one of the leading causes of cancer-related deaths in the world. There were about 608,000 deaths from CRC in the world, accounting for $8 \%$ of all cancers in 2008 (Ferlay et al. 2010). The main cause for the high mortality rate is that CRC remains highly undiagnosed. A vast amount of literatures have been reported for the prevention of CRC (Goh 2013; Temraz et al. 2013; Madka and Rao 2013; Ishii et al. 2013; Suchanek et al. 2014). Colon carcinogenesis involves a number of etiological factors. Therefore, effective preventive strategies and molecular targets need to be identified. Lifestyle risk factors are modulated during colon carcinogenesis and can be targeted for CRC prevention (Gingras and Beliveau 2011; Derry et al. 2013). There is also evidence to support that long-term sedentary work increases the risk of CRC (Howard et al. 2008; Boyle et al. 2011). However, the precise molecular mechanism or the cause of CRC by the sedentary lifestyle has not been clarified.
Genomic technologies offer the great promise of a comprehensive understanding of CRC. These technologies have been applied to characterize the cancer at the molecular level and identified a plethora of new molecular markers. These markers have contributed a great deal to the understanding of the molecular mechanisms causing the CRC. For instance, Hv1 voltage-gated proton channels mediate rapid and selective transmembrane $\mathrm{H}+$ flux, and are gated by both voltage and $\mathrm{pH}$ gradients (Ramsey et al. 2010). Hv1 expression increased in CRC tissues and significantly associated with the tumor size, classification, lymph node status, and clinical stage. Thus, Hv1 may be a potential biomarker for the diagnosis and prognosis of CRC (Wang et al. 2013b). Another example, matrix remodeling associated 5 (MXRA5, one of the matrix-remodeling associated proteins) is thought to be involved in cell adhesion and signaling, which is important for cell fusion (Wang et al. 2013a). In CRC tissues, MXRA5 is significantly expressed compared with their normal adjacent mucosa. Therefore, MXRA5 has potential value in early detection of CRC and prediction of omental metastasis

Received December 12, 2013; revised and accepted February 13, 2014. Published online March 12, 2014; doi: 10.1620/tjem.232.167. Correspondence: Qin Lu, Department of Gastroenterology, Affiliated Zhongda Hospital of Southeast University, 87\# Dingjiaqiao Road, Nanjing, Jiangsu 210009, P.R. China. e-mail: qinlunj@163.com 
(Wang et al. 2013a). In contrast, protein inhibitor of activated signal transducer and activators of transcription 3 (PIAS3, an E3-type small ubiquitin-like modifier ligase), as a novel biomarker, has a role in controlling the development of malignancy, which is expressed at a lower level in most common malignancies compared with corresponding normal tissues (Dagvadorj et al. 2010; Dunnebier et al. 2010; Dabir et al. 2014). PIAS3 is also a potential biomarker target for early cancer detection and therapy of CRC (Li et al. 2013). For a final example, DEK (The protein binds to cruciform and superhelical DNA and induces positive supercoils into closed circular DNA and is involved in splice site selection during mRNA processing) is related to chromatin reconstruction and gene transcription, and plays an important role in cell apoptosis (Hua et al. 2009). High expression levels of the human DEK gene have been correlated with numerous human malignancies (Privette Vinnedge et al. 2013). DEK also plays an important role in the progression of CRC and is an independent poor prognostic factor of CRC (Lin et al. 2013). All these biomarkers offer a great convenience in the diagnosis of CRC.

Continued focus on primary prevention of $\mathrm{CRC}$, in combination with lifestyle and molecular mechanisms, will be vital in attaining the greatest progress against the disease. Thus, it is of great clinical interest to explore the relationship between the sedentary time and protein levels of the biomarkers of Hv1, MXRA5, PIAS3 and DEK.

\section{Materials and Methods}

\section{Participants}

The study of human subjects and informed consent documents were approved by the Human Research Ethic Committee from Affiliated Zhongda Hospital of Southeast University (Nanjing, China). In addition, the data were collected under a Certificate of
Confidentiality obtained from the same university. From March 7th, 2010 to May 6th, 2012, a total of 228 CRC patients were recruited by Affiliated Zhongda Hospital of Southeast University. In the same period, 80 healthy volunteers were also recruited. To avoid the biased results, all the controls and patients were from the same city (Nanjing, China). All the patients and healthy subjects were unrelated ethnic Han Chinese. All the subjects were with the similar cultural and economic background.

The diagnostic criteria for $\mathrm{CRC}$ were used according to a previous report (Bouwens et al. 2013). Endoscopic submucosal dissection (ESD) is accepted as a minimally invasive treatment for gastric cancer, but it is not widely used to treat the colorectum because of technical difficulty. Sinker-assisted ESD is effective for the removal of selected superficial CRC (Saito et al. 2005). Early CRC limited to the mucosa or with slight submucosal invasion are candidates for endoscopic resection (Yokoyama et al. 2002). ESD can be used for the examination of colorectal lesions, depressed lesions and laterally spreading tumors of non-granular type.

Sinker-assisted ESD is effective for the removal of superficial colorectal tissues, making it now the preferred approach in obtaining the surgical specimens of healthy volunteers. Considering ethical issues is important especially for healthy volunteers. The experts of Sinker-assisted ESD explained healthy volunteers the importance of voluntary participation, the privacy handling, and the potential risk before obtaining informed consent. The health of the volunteers would be checked in the following half year. No injury was found after all surgical operation. However, the experts did not persuade any participant to donate his or her minus tissues if he or she casted some doubts on the safety of the techniques. For all healthy subjects, they had normal colorectal ESD and were free of the tumor symptoms.

The patients with $\mathrm{CRC}$ and healthy subjects were stratified in four groups according to the sedentary time (Table 1). In the study, we examined 228 adults (aged 50-65 years) who reported to be diagnosed with CRC within one year and not any other cancer, or cardiovascular disease, or inflammation disease. All the patients were identified in Affiliated Zhongda Hospital of Southeast University.

Table 1. The characteristics of the patients with CRC and healthy subjects.

\begin{tabular}{|c|c|c|c|c|c|c|c|c|}
\hline & & & Healthy & Stage 0 & Stage I & Stage II & Stage III & Stage IV \\
\hline \multirow{6}{*}{$\frac{0}{\sum \pi}$} & \multicolumn{2}{|l|}{ Cases } & 48 & 39 & 29 & 27 & 20 & 13 \\
\hline & \multicolumn{2}{|l|}{ Age } & $57.5 \pm 6.8$ & $56.7 \pm 5.9$ & $57.3 \pm 5.5$ & $56.4 \pm 6.3$ & $57.1 \pm 7.0$ & $58.3 \pm 7.3$ \\
\hline & \multicolumn{2}{|l|}{ BMI $\left(\mathrm{kg} / \mathrm{m}^{2}\right)$} & $24.7 \pm 5.1$ & $25.8 \pm 5.7$ & $26.5 \pm 6.1$ & $27.3 \pm 6.6$ & $28.9 \pm 7.1$ & $29.7 \pm 7.7$ \\
\hline & \multicolumn{2}{|c|}{ Daily calorie intake (kcal) } & $2,238 \pm 356$ & $2,169 \pm 412$ & $2,316 \pm 398$ & $2,264 \pm 379$ & $2,145 \pm 321$ & $2,195 \pm 432$ \\
\hline & \multirow{2}{*}{$\begin{array}{l}\text { Sedentary time (h/d) } \\
\mathrm{L} / \mathrm{N} / \mathrm{M} / \mathrm{H}\end{array}$} & TV viewing & $7 / 20 / 13 / 8$ & $5 / 15 / 12 / 6$ & $2 / 10 / 9 / 8$ & $2 / 7 / 10 / 8$ & $1 / 5 / 7 / 7$ & $1 / 2 / 4 / 6$ \\
\hline & & Sitting at work & 9/19/11/9 & $5 / 16 / 9 / 7$ & $1 / 9 / 10 / 9$ & $1 / 7 / 9 / 10$ & $1 / 4 / 8 / 7$ & $0 / 1 / 7 / 5$ \\
\hline \multirow{6}{*}{ 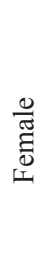 } & \multicolumn{2}{|l|}{ Cases } & 32 & 30 & 25 & 18 & 15 & 12 \\
\hline & \multicolumn{2}{|l|}{ Age } & $56.9 \pm 6.5$ & $57.1 \pm 6.9$ & $57.8 \pm 6.3$ & $57.7 \pm 6.6$ & $58.1 \pm 7.3$ & $58.2 \pm 7.0$ \\
\hline & \multicolumn{2}{|l|}{ BMI $\left(\mathrm{kg} / \mathrm{m}^{2}\right)$} & $24.1 \pm 4.8$ & $25.1 \pm 5.9$ & $26.2 \pm 6.8$ & $26.8 \pm 6.8$ & $28.2 \pm 7.9$ & $28.9 \pm 8.0$ \\
\hline & \multicolumn{2}{|c|}{ Daily calorie intake (kcal) } & $1,945 \pm 273$ & $1,997 \pm 302$ & $1,882 \pm 277$ & $1,899 \pm 311$ & $1,832 \pm 251$ & $1,849 \pm 297$ \\
\hline & \multirow{2}{*}{$\begin{array}{l}\text { Sedentary time }(\mathrm{h} / \mathrm{d}) \\
\mathrm{L} / \mathrm{N} / \mathrm{M} / \mathrm{H}\end{array}$} & TV viewing & $5 / 17 / 7 / 3$ & $4 / 12 / 8 / 6$ & $3 / 10 / 6 / 6$ & $2 / 6 / 5 / 5$ & $1 / 2 / 6 / 6$ & $0 / 1 / 5 / 6$ \\
\hline & & Sitting at work & $4 / 19 / 6 / 3$ & $5 / 11 / 9 / 5$ & $2 / 10 / 8 / 6$ & $1 / 4 / 7 / 6$ & $0 / 2 / 6 / 7$ & $0 / 0 / 5 / 7$ \\
\hline
\end{tabular}

The CRC has the five stages, 0 , I, II, III and IV. The sedentary time is marked as L if the time spent being sedentary is less than 1 $\mathrm{h} / \mathrm{d}$. In the same way, $1-3 \mathrm{~h} / \mathrm{d}$ is marked as $\mathrm{N}, 4-6 \mathrm{~h} / \mathrm{d}$ as $\mathrm{M}$ and more than $6 \mathrm{~h} / \mathrm{d}$ as $\mathrm{H}$. The cases for the sedentary lifestyles are showed in the order $\mathrm{L} / \mathrm{N} / \mathrm{M} / \mathrm{H}$. d, day. 
Eligible cases were all Nanjing residents who had been diagnosed with histological confirmation. Patients with a history of previous CRC were excluded. The final stage of the CRC was determined according to the general rules for TNM classification system of tumors by International Union Against Cancer (UICC) (Sobin et al. 2011). There are five stages for CRC (Stage 0, I, II, III and IV).

\section{Assessment of sedentary time}

The sedentary time was assessed according to a previous report (Matthews et al. 2012). The following 2 distinct exposures were considered: television viewing and sitting at work. The time for television viewing was assessed by the question, "During a typical 24-hour period over the past 10 years before the diagnosis, how much time did you spend watching television (Less than 1, 1-3, 4-6, more than $6 \mathrm{~h}$ /day)?" To assess the time for sitting at work, participants were asked, "During a typical 24-hour period over the past 10 years before the diagnosis, how much time did you spend occupational sitting (Less than 1, 1-3, 4-6, more than $6 \mathrm{~h}$ /day)?" The time spent being sedentary is marked as $\mathrm{L}$ if it is less than $1 \mathrm{~h} /$ day. In the same way, $1-3 \mathrm{~h}$ /day is marked as $\mathrm{N}, 4-6 \mathrm{~h} /$ day as $\mathrm{M}$ and more than $6 \mathrm{~h}$ /day as $\mathrm{H}$.

\section{Potential confounding and mediating factors}

Potential confounding and mediating factors were identified in the associations between sedentary lifestyles and adiposity, which might be closely related with the risk of CRC (Bardou et al. 2013; Guffey et al. 2013; Vermorken et al. 2014). During the survey, the body mass index (BMI, weight in kilograms divided by height in square meters) was measured. Gender difference has been reported to be related with the risk of CRC (Tan et al. 2013; Ritvo et al. 2013). Here, the data for female and male in different stages of CRC were collected. Tabaco smoking and alcohol consumption can cause the risk of CRC (Acott et al. 2008; Zhao et al. 2010; Crous-Bou et al. 2013; Peng et al. 2013). To avoid the interference, all individuals were never smokers or alcohol drinkers. To avoid age-related issues, all subjects aged between 50 and 65 years old. All participants reported food consumption frequency was at 3/day. The food consumption was adhered to a healthy nordic food index as previously reported (Kyro et al. 2014). Calorie intake was assessed according to a previous report ( $\mathrm{Li}$ et al. 2012).

\section{ELISA assays}

The concentration of proteins was determined using ELISA kits. ELISA assays were performed according to the manufacturer's instructions (Hv1 ELISA kit, No. ABIN423447, Biotechnology (Group) Co., Ltd., Shanghai, China; MXRA5 ELISA kit, No. CSBEL015255HU, Wuhan Hi-tech Medical Devices Park, Wuhan, China; DEK ELISA kit, No. E98770Hu, Uscn Life Science Inc., Wuhan, China; PIAS3 ELISA kit, No. CSB-E10009h, Shanghai Gaochuang Chemical technology Co., Ltd., Shanghai, China). Biopsy specimen was ground in a small amount of sterile buffer. A 1/200 dilution of specimen in buffer was introduced in specific antibody-coated microtiter wells and incubated at room temperature for $30 \mathrm{~min}$. The wells were washed with PBS for three times, and a peroxidase conjugated anti-human IgG was added and incubated for another $30 \mathrm{~min}$. After washing, tetramethylbenzidine (Donglinchangsheng Biotechnology Co., Ltd., Beijing, China) was added and incubated for $10 \mathrm{~min}$. The optical density was measured at $450 \mathrm{~nm}$. The concentration was determined according to a calibrator curve.
Real-time quantitative reverse transcription polymerase chain reaction (qRT-PCR)

The expression levels of Hv1, MXRA5, PIAS3 and DEK mRNAs were estimated using real-time qRT-PCR. Firstly, total RNA was isolated from the CRC tissues using Catrimox-14 TM RNA Isolation Kit Ver.2.11 (Takara Biotechnology (Dalian) Co., Ltd., Dalian, China). Genomic DNA was digested with recombinant DNase I (Takara Biotechnology (Dalian) Co., Ltd., Dalian, China). Before RNA amplification, denaturing agarose gel electrophoresis and A260/A280 measurement were used to estimate the integrity and purity of RNA.

An initial strand of cDNA was synthesized from $500 \mathrm{ng}$ of RNA using the random primer. Two microliters of cDNA were used as template for the real-time PCR detection of the Hv1, MXRA5, PIAS3 and DEK transcripts in a $25-\mu 1$ reaction SYBR green (Roche, Mannheim, Germany) on a 96-well-plate using Lightcycler480 (Roche). The primers for real-time qRT-PCR were: Hv1 (GenBank No.: NM_032369.3), 5'-CTTCAGGGGCATGTTGAGGA-3' and 5'-GTTGCCGTTCTGAACGTGTC-3'; MXRA5 (GenBank No.: NM_015419.3), Sense primer, 5'-GTTAAGAAGCCTGCGGAA AC-3' and Antisense primer, 5'-GAGTGCTCATGGTGGATGAC-3'; PIAS3 (GenBank No.: CR457090.1), Sense primer, 5'-TCGACT CTCAGCCACTGTTC-3' and Antisense primer, 5'-ACTGCCTCA CCAGGTACACA-3'; DEK (GenBank No.: NM_003472.3), Sense primer, 5'-GTTAAAGAGCATCTGTGAGG-3' and Antisense primer, 5'-CATCTGTAGGGGGTTTCTTC-3'; and glyceraldehyde-3-phosphate dehydrogenase (GAPDH), 5'-CCCTTCATTGACCT CAACTAC-3' and 5'-CCACCTTCTTGATGTCATCAT-3'. GAPDH was used as an internal control. All genes were amplified by 40 cycles of heating for $30 \mathrm{~s}$ at $94^{\circ} \mathrm{C}$, followed by $1 \mathrm{~min}$ at $60^{\circ} \mathrm{C}$. The quality of the synthesized cDNA was verified via the reference gene GAPDH. The data represented the average transcriptional levels of target genes, relative to GAPDH, from three independent samples.

\section{Measurement of the expression levels of Hv1, MXRA5, PIAS3 and} DEK proteins

Surgical specimen samples were obtained from all subjects and stored at $-80^{\circ} \mathrm{C}$ immediately. The levels of Hv1, MXRA5, PIAS3 and DEK were examined by western blot. Total protein extracts, from selected CRC tissues, were separated on 12\% SDS-PAGE gradient gels and followed by electrotransfering to PVDF membranes (Bio-Rad). The membranes were blocked ( $5 \%$ dry milk, $0.5 \%$ Tween $20,1 \times$ TBS, $0.1 \mathrm{M}$ Tris- $\mathrm{HCl}, 0.5 \mathrm{M} \mathrm{NaCl}$ ) for $1 \mathrm{~h}$, incubated with the primary antibody diluted 1:500 in blocking buffer, and washed in Tris-buffered saline supplemented with Tween 20. The secondary horseradish peroxidase-conjugated antibody (anti-mouse immunoglobulin/horseradish peroxidase, Amersham, Braunschweig, Germany) was diluted 1:3,000 in blocking buffer, and chemiluminescence detection was carried out using a Chemidoc charge-couple device camera (Bio-Rad).

\section{Statistical analysis}

Forced-entry linear regression model was used to examine the associations of the levels of Hv1, MXRA5, PIAS3 and DEK levels, and the sedentary time, or BMI or calorie intake of the patients with CRC. Data were analyzed using Statview 5.0 software (Abacus Systems, Berkeley, CA, USA). The $P$ value was accepted as significant when it was $<0.05$. 


\section{Results \\ Effects of sedentary time on BMI}

Here, 228 CRC patients (128 males, $57.8 \pm 7.8$ years; 100 females, $57.7 \pm 7.5$ years) and 80 healthy subjects ( 48 males, $57.5 \pm 6.8$ years; 32 females, $56.9 \pm 6.5$ years) were recruited. Considering the potential confounding factors for causing the risk of CRC, the gender and age were not the contributing factors (Table 1) $(P>0.05)$. Many other factors were excluded during the data collection. The study found the associations between sedentary time and BMI. The BMI was higher when the sedentary time was longer (Table 1) $(P<0.01)$. Both watching TV and sitting at work were incrementally detrimental to BMI.

\section{The effects of sedentary time on the risk of CRC}

The analyses for the effects of sedentary time on the risk of CRC were undertaken for the studies in Table 1. All the CRC patients were diagnosed by professional doctors. The results showed that 69 cases of stage 0,54 cases of stage I, 45 cases of stage II, 35 cases of stage III and 25 cases of stage IV. The stages of CRC were closely related with the sedentary time. A statistically significant increase in the risk of CRC was seen in watching TV and sitting at work in both males and females. In the healthy subjects and the CRC patients at stage 0 , the less and normal sedentary were the major proportions among the four types of sedentary time (Table 1$)(P<0.01)$. In the patients at stage III and IV, more and heavy sedentary were the major proportions (Table 1$)(P<0.01)$. The daily sedentary time is more than $6 \mathrm{~h} / \mathrm{d}$ in the most of CRC patients at stage IV. Someone spent almost $16 \mathrm{~h}$ each day when the time for reviewing TV and sitting at work was combined (Fig. 1). To reduce the other factors interference, the similar ages between CRC patients and healthy subjects were chosen. Comparatively, the healthy subjects spent less time for viewing television and sitting at work each day (Fig. 1). The sedentary time in healthy subjects was significantly shorter than that in the CRC patients. The results suggested that sedentary lifestyles were associated with the risk of CRC.

The mRNA levels of Hv1, MXRA5, PIAS3 and DEK in CRC patients

The quantitative RT-PCR analysis showed that the mRNA levels of Hv1, MXRA5 and DEK were higher when the CRC patients were at advanced stage. Inversely, the mRNA level of PIAS3 was lower when the CRC patients were at advanced stage. The levels of Hv1, MXRA5 and DEK were the highest while the level of PIAS3 was the lowest when CRC patients were at stage IV (Fig. 2).

The protein levels of Hv1, MXRA5, PIAS3 and DEK in CRC patients

The concentration of Hv1 is closely related with the development of CRC (Wang et al. 2013b). The average

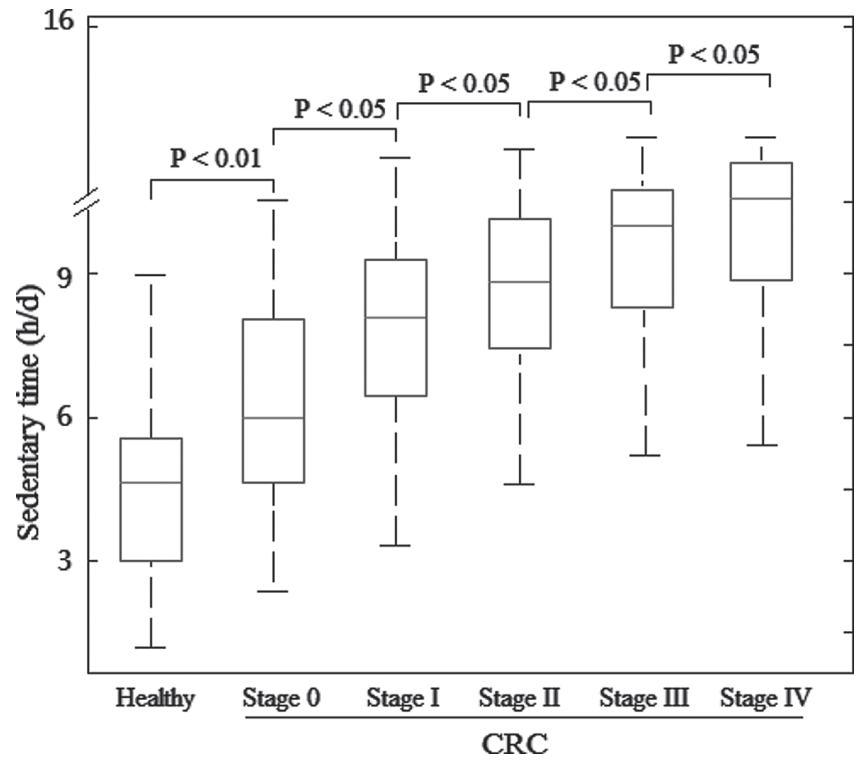

Fig. 1. Box plots of sedentary time of healthy subjects and CRC patients.

CRC patients were divided into five groups according to the development of CRC: stage 0, I, II, III and IV. The sedentary time presented the time for reviewing TV and sitting at work, both of which were combined. The red bars in the boxes were average sedentary time and the boxes represented $95 \%$ of the samples. The cases for the study are as follows: $n=80$ for healthy subjects, and $n=$ $69,54,45,35$ and 25 for CRC stage 0, I, II, III and IV respectively. The error bars were above or below the boxes.

concentration of Hv1 could reach the highest level when the CRC developed to the stage IV (Figs. 3 and 4). Thus, the result was consistent with a previous report that Hv1 was a potential biomarker for the diagnosis and prognosis of CRC, and a potential target for anticancer drugs in CRC therapy (Wang et al. 2013b). MXRA5 is highly expressed in CRC tissues (Wang et al. 2013a). Here, we found that the concentration of MXRA5 was positively related with the development of CRC. Just as Hv1, the average concentration of MXRA5 could reach the highest level when the CRC patients were at the stage IV (Figs. 3 and 4). PIAS3 can affect the growth of cancer cells in some malignancy (Ogata et al. 2006; Liu et al. 2011). Here, we found that the level of PIAS3 was lower in CRC patients were at advanced stage. The average concentration of PIAS3 reached the lowest level when the CRC patients were at stage IV (Figs. 3 and 4). The DEK is also associated with human carcinogenesis (Wise-Draper et al. 2006). Here, we found that the protein level of DEK was higher when the CRC patients were at advanced stage (Figs. 3 and 4), which was consistent with a previous report that DEK played an important role in the progression of CRC and it was an independent poor prognostic factor of CRC (Lin et al. 2013). 


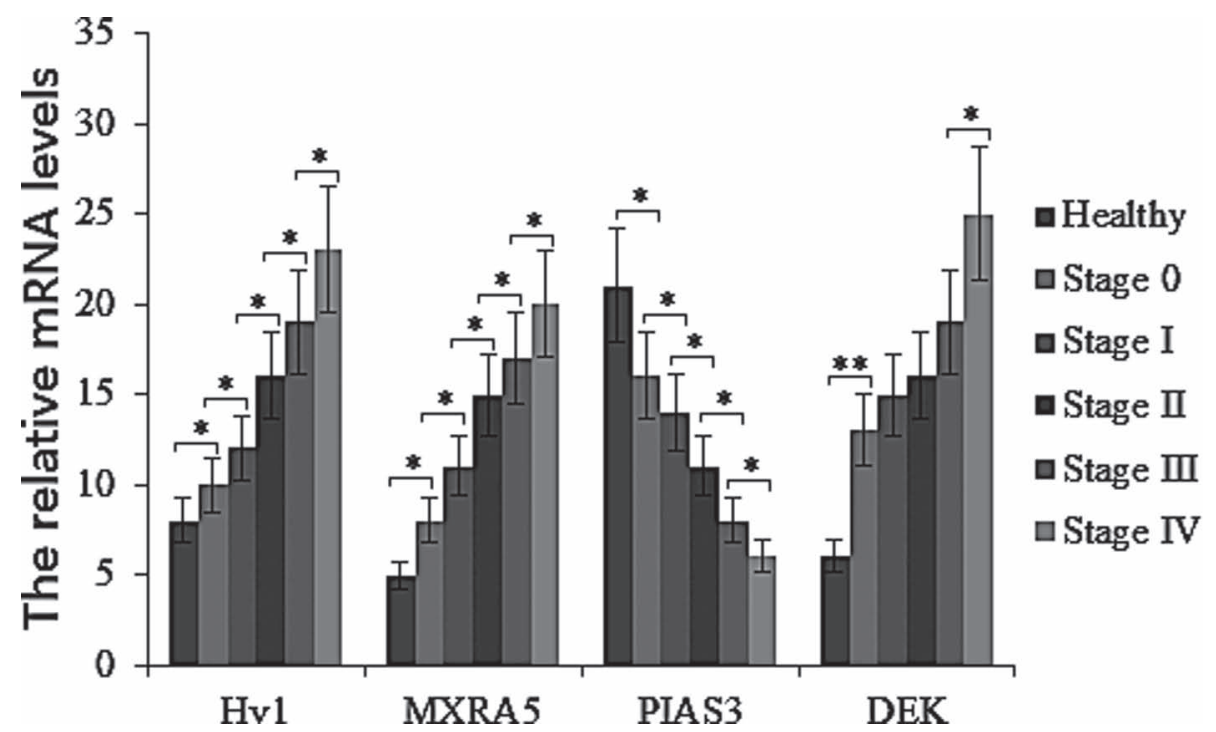

Fig. 2. The mRNA levels of CRC biomarkers in healthy subjects and CRC patients.

Five groups were divided according to the developing stages of CRC (Stage 0, I, II, III and IV). The cases for the study are as follows: $n=80$ for healthy subjects, and $n=69,54,45,35$ and 25 for CRC stage 0 , I, II, III and IV respectively. Each bar represented the mean \pm S.D. of three independent experiments. $* P<0.05$ and $* * P<0.01$ compared to a previous value.
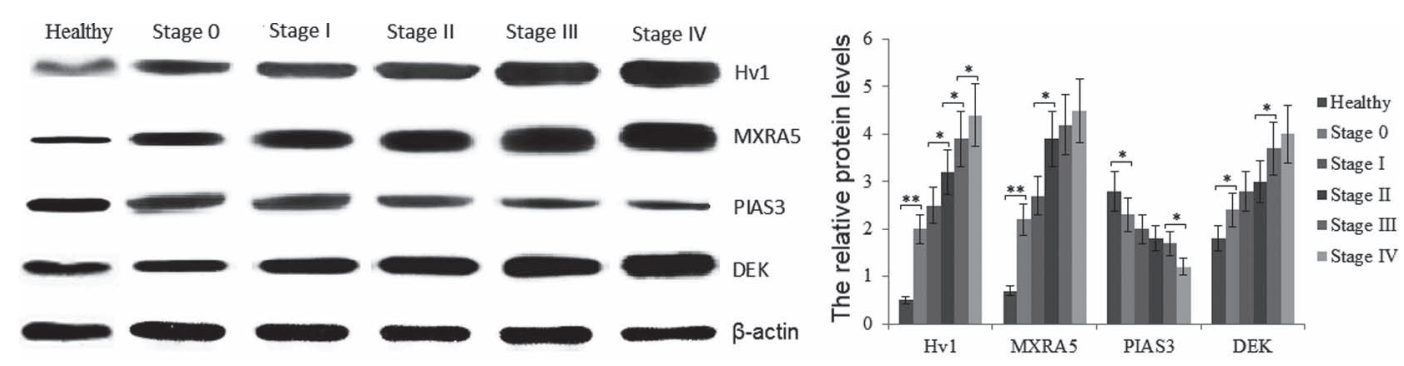

Fig. 3. The protein levels of CRC biomarkers in healthy subjects and CRC patients.

Five groups were divided according to the developing stages of CRC (Stage 0, I, II, III and IV). The cases for the study are as follows: $n=80$ for healthy subjects, and $n=69,54,45,35$ and 25 for CRC stage 0 , I, II, III and IV respectively. Each bar represented the mean \pm S.D. of three independent experiments. $* P<0.05$ and $* * P<0.01$ compared to a previous value.

The association between the concentrations of $H v 1$, MXRA5, PIAS3 or DEK and daily calorie intake or BMI

We hypothesized that the concentrations of Hv1, MXRA5, PIAS3 or DEK might be affected by daily calorie intake or BMI, so we investigated the association between the concentrations of Hv1, MXRA5, PIAS3 and DEK, and daily calorie intake or BMI. Our observations revealed the values of daily calorie intake did not affect the levels of Hv1, MXRA5, PIAS3 and DEK (all $P>0.05$ ) (Fig. 5A, B, $\mathrm{C}$ and $\mathrm{D})$. The results suggested that daily calorie intake might not be associated with the risk of CRC. Comparatively, the association between the values of BMI and the levels of Hv1, MXRA5, PIAS3 or DEK in CRC was statistically significant (all $P<0.05$ ) (Fig. 5E, F, G and H). All the results implied that daily calorie intake did not affect the BMI, which was consistent with a previous report: reduced calorie intake and frequent use of lowcalorie food products could not decrease the prevalence of obesity (Heini and Weinsier 1997). However, the sedentary time might be related with the development of CRC via regulating BMI, which could affect the levels of Hv1, MXRA5, PIAS3 and DEK since the values of BMI were closely related with the levels of the four biomarkers of CRC (Fig. 5E, F, G and H).

The association between the concentrations of $H v 1$, MXRA5, PIAS3 and DEK, and the sedentary time

We hypothesized that CRC might be caused by the sedentary lifestyles regulating the levels of Hv1, MXRA5, PIAS3 and DEK, and we investigated whether sedentary time affected the expression levels of Hv1, MXRA5, PIAS3 and DEK in all subjects. In the CRC patients, our observations revealed the influence of sedentary time on the levels of Hv1, MXRA5, PIAS3 and DEK in the CRC tissue (all $P$ $<0.05$ ) (Figs. 4 and 6). The association between the sedentary time and the levels of Hv1, MXRA5, PIAS3 or DEK in the CRC tissues was statistically significant (Fig. 6). With the time prolonging from less- to heavy-sedentary, the 

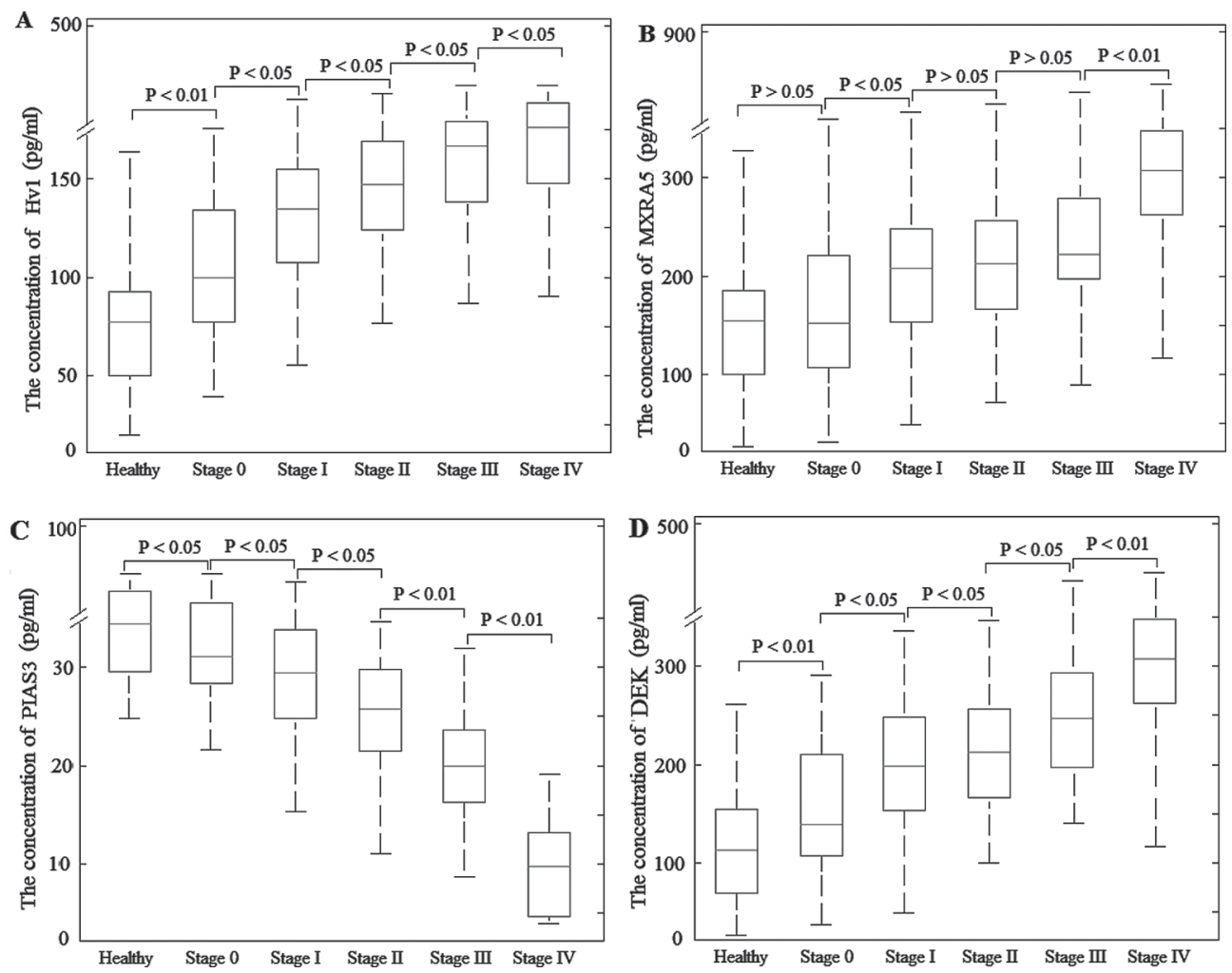

Fig. 4. Box plots of the concentration of CRC biomarkers in healthy subjects and CRC patients.

The concentration was analyzed via ELISA. A, the concentration distribution of Hv1 in all subjects. B, the concentration distribution of MXRA5 in all subjects. C, the concentration distribution of PIAS3 in all subjects. D, the concentration distribution of DEK in all subjects. The cases for the study are as follows: $n=80$ for healthy subjects, and $n=69,54,45,35$ and 25 for CRC stage 0, I, II, III and IV respectively. The bars in the boxes were average concentration and the boxes represented $95 \%$ of the samples. The error bars were above or below the boxes. $* P<0.05$ and $* * P<0.01$, compared to a previous value.

levels of Hv1, MXRA5 and DEK were higher in the CRC patients of advanced stage $(P<0.05)$ (Fig. 6A, B and D, left panels). Inversely, the level of PIAS3 was lower in CRC patients of advanced stage $(P<0.05)$ (Fig. 6C, left panel). Comparatively, the levels of Hv1, MXRA5, PIAS3 and DEK had no significant change in all healthy subjects with the change of sedentary lifestyles $(P>0.05)$ (Fig. 6A, $\mathrm{B}, \mathrm{C}$ and $\mathrm{D}$, right panels). All the results implied that the sedentary lifestyles were closely related with the development of CRC via regulating the levels of Hv1, MXRA5, PIAS3 and DEK.

\section{Discussion}

$\mathrm{CRC}$ is the third most common cancer worldwide with over 1 million new cases diagnosed each year. Advances in treatment and survival are likely to have increased lifetime costs of managing the disease (Ó Céilleachair et al. 2013). A previous report shows that long-term sedentary work may increase the risk of CRC (Boyle et al. 2011). A sedentary lifestyle, long believed to contribute to chronic conditions such as heart disease, can increase the risk of CRC. Physically active individuals have a lower risk of developing CRC. Recreational physical activity after the diagnosis of stages I to III CRC may reduce the risk of
CRC-specific and overall mortality (Meyerhardt et al. 2006). Activities are considered if they require very low energy expenditure, such as prolonged sitting. Occupations requiring physical activity were associated with a $44 \%$ reduced risk of CRC compared with those requiring long siting (Boyle et al. 2011). Physical inactivity and high BMI have been linked to increased risk of CRC. Recommendations to increase physical activity and maintain lean body weight should receive greater emphasis as part of a feasible approach to the prevention of CRC (Martínez et al. 1997).

However, the molecular mechanism for causing the disease is still elusive. Finding suitable biomarker for CRC is important for exploring the molecular mechanism. However, the levels of some important proteins cannot be detected in serum sometimes, which might be affected by the human clinical and metabolic condition. Thus, only submitted CRC specimens were only considered here.

Hv1 mediates NADPH oxidase function by compensating cellular loss of electrons with protons (Ramsey et al. 2009) and can be highly expressed in CRC tissues (Wang et al. 2013b). The protein MXRA5 contains 7 leucine-rich repeats and 12 immunoglobulin-like C2-type domains related to perlecan. MXRA5 is the second most frequently mutated gene in non-small cell lung carcinomas 

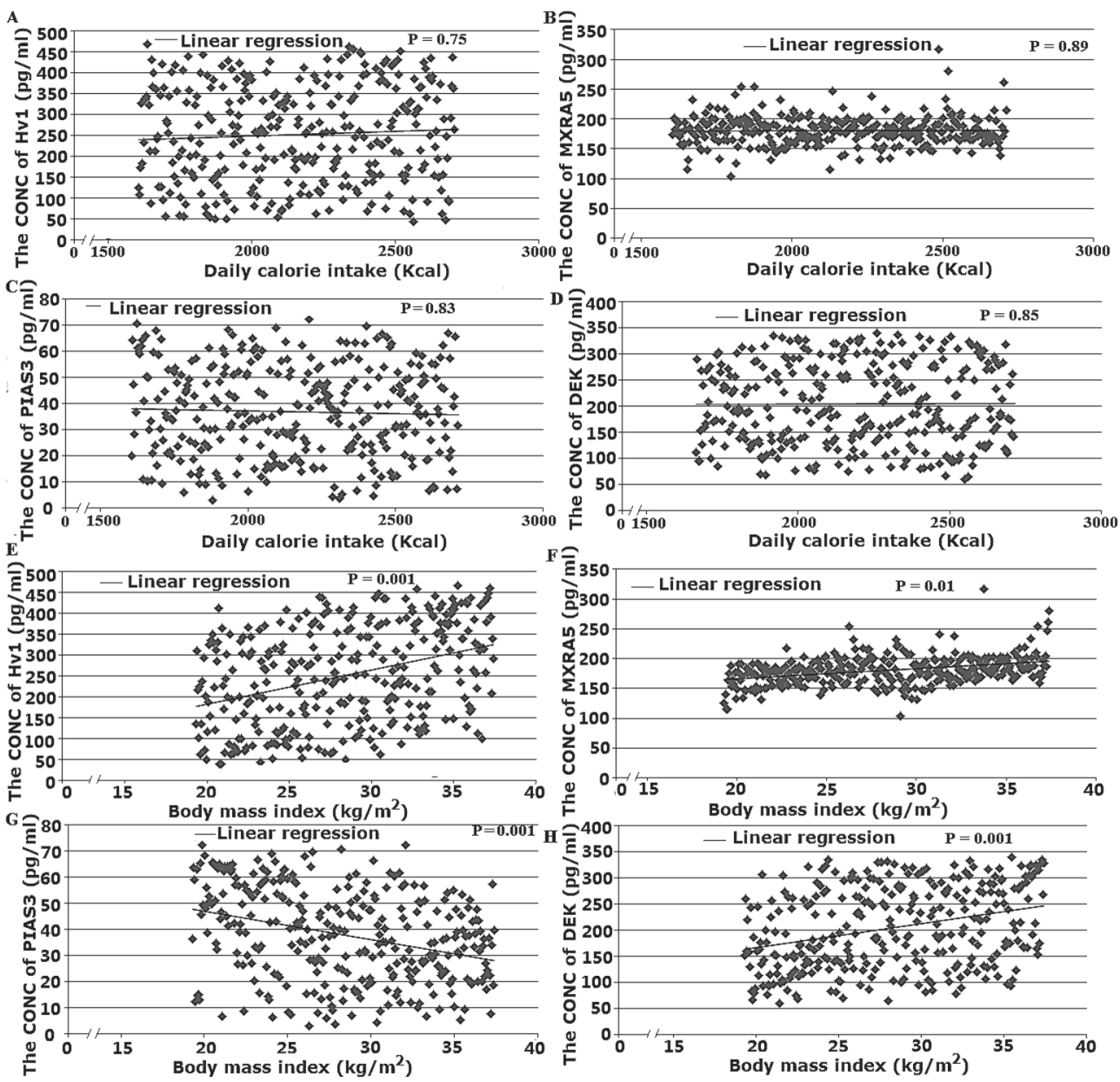

Fig. 5. The association between the concentrations of CRC biomarkers and daily calorie intake or BMI.

All the concentrations were analyzed via ELISA. A, the association between the concentrations of Hv1 and the values of daily calorie intake. B, the association between the concentrations of MXRA5 and the values of daily calorie intake. $\mathrm{C}$, the association between the concentrations of PIAS3 and the values of daily calorie intake. D, the association between the concentrations of DEK and the values of daily calorie intake. E, the association between the concentrations of $\mathrm{Hv} 1$ and the values of BMI. F, the association between the concentrations of MXRA5 and the values of BMI. G, the association between the concentrations of PIAS3 and the values of BMI. H, the association between the concentrations of DEK and the values of BMI. The cases for the study are as follows: $n=80$ for healthy subjects and $n=228$ for CRC patients. The $P$ value showed the statistical significance for the concentration change of four molecules when the values of daily calorie intake or BMI changed. The spearman's rank correlation was showed by rho- and $P$-value. The rhovalue and $P$-value were showed above the $\mathrm{x}$-axis. The rho value represented the positive or negative correlation between the concentrations of the four biomarkers and the values of daily calorie intake or BMI. The result was considered statistically significant if $P<0.05$. CONC, concentration.

(Xiong et al. 2012). MXRA5 is highly expressed in CRC tissues, and has potential value in early detection of CRC (Wang et al. 2013a). PIAS3 is an endogenous inhibitor of STAT3 and has antiproliferative properties. Glioblastoma and squamous cell lung cancer lack PIAS3 expression (Dabir et al. 2011). PIAS 3 can affect the growth of cancer cells by inhibiting the JAK/STAT and PI3-K/Akt signaling pathways or regulating its SUMO (small-ubiquitin like modifiers) ligase activity in some malignancy (Liu et al. 2011). DEK protein is related to chromatin reconstruction and gene transcription, and plays an important role in cell apoptosis. DEK is associated with human carcinogenesiseither as a fusion with the CAN nucleoporin protein or when transcriptionally up-regulated (Wise-Draper et al. 2006). Thus, we considered the four kinds of important molecules which were closely associated with the risk of CRC.

The CRC tissues sampling can increase the detection of tumor cells and used for the detection of mRNA levels. The mRNA levels regulate protein synthesis and also reflect the levels of protein synthesis. Thus, the mRNA levels of Hv1, MXRA5, PIAS3 and DEK were examined firstly, and then the protein levels were determined. The mRNA and protein levels tended to change in parallel with the development of CRC (Figs. 2 and 3). Hv1, MXRA5, PIAS3 and DEK could be target molecules for drug therapy, 

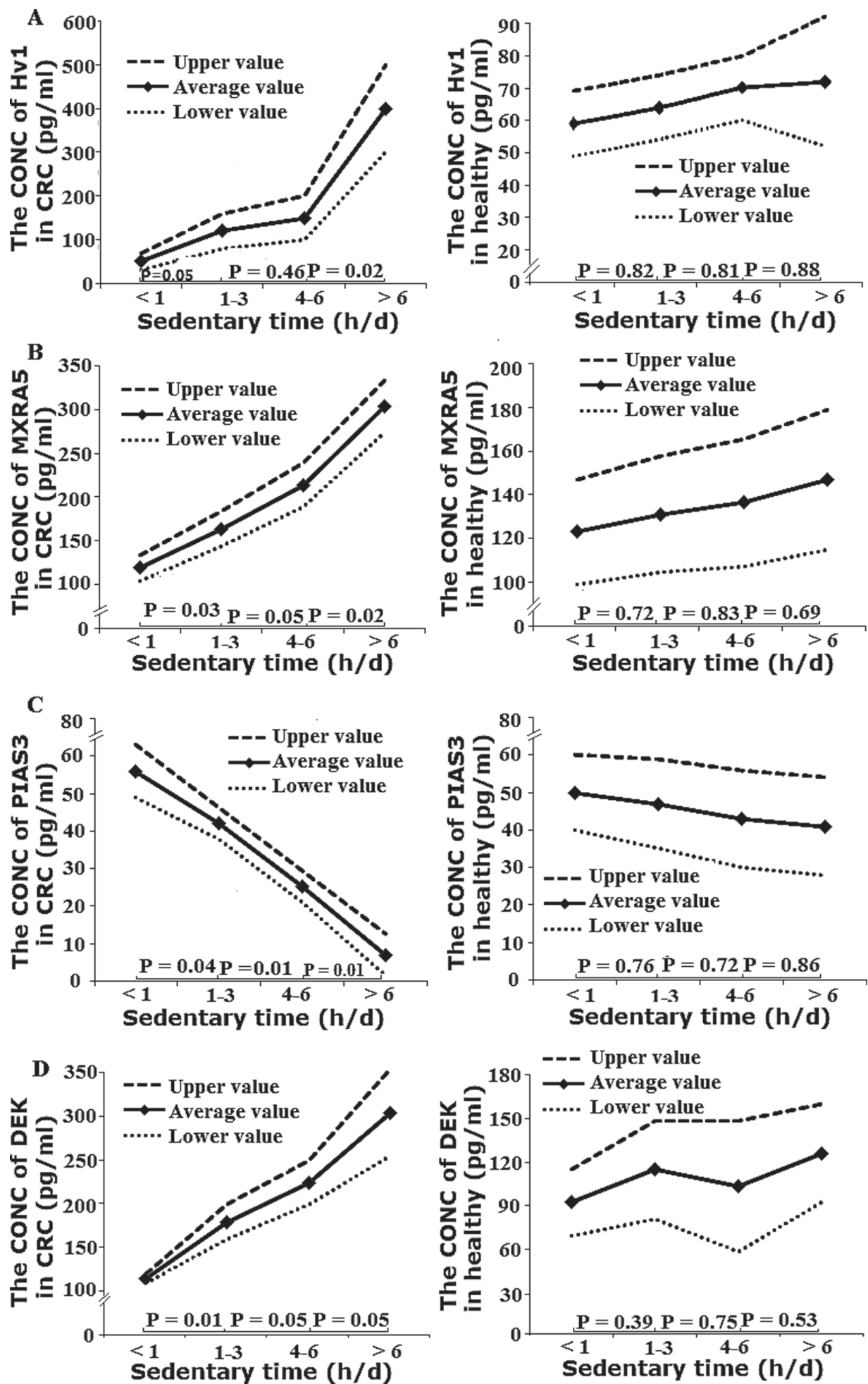

Fig. 6. The association between the concentrations of CRC biomarkers and sedentary time.

All the concentrations were analyzed via ELISA. A, the association between the concentrations of Hvl and daily sedentary time. B, the association between the concentrations of MXRA5 and daily sedentary time. C, the association between the concentrations of PIAS3 and daily sedentary time. D, the association between the concentrations of DEK and daily sedentary time. Left panel showed the association between the concentrations of the molecules and daily sedentary time in CRC patients. Right panel showed the association between the concentrations of the molecules and daily sedentary time in healthy subjects. The cases for the study are as follows: $n=80$ for healthy subjects and $n=228$ for CRC patients. Statistical analysis was done by $t$-test. The $P$ value showed the statistical significance for the concentration change of four molecules when the sedentary lifestyles changed. The $T$-value and $P$-value were showed above the $\mathrm{x}$-axis. The result was considered statistically significant if $P<0.05$. CONC, concentration. 
which is consistent with previous reports (Wang et al. 2013a, b; Li et al. 2013; Lin et al. 2013). Furthermore, all the biomarkers are averagely higher or lower than those in healthy subjects (Fig. 2). Thus, the development of CRC may be associated with the changes in the expression levels of Hv1, MXRA5, PIAS3 or DEK. The levels of these biomarkers could not reflect the disease severity but might be related with the development of CRC, because high concentration of Hv1, MXRA5 or DEK, or the low concentration of PIAS3 was also detected in healthy subjects (Fig. 1). Because of this, the levels of four biomarkers should be considered together.

Since the expression levels of Hv1, MXRA5, PIAS3 or DEK were higher or lower in the CRC patients, the changed levels of these biomarkers may also be the result of an indirect mechanism trigger by long-term sedentary. The levels of Hv1, MXRA5, PIAS3 and DEK were significantly affected by the length of sedentary time, suggesting long-term sitting increases the risk of hospitalization for CRC by affecting the levels of the biomarkers, Hv1, MXRA5, PIAS3 and DEK. The association between the length of sedentary time and the levels of Hv1, MXRA5, PIAS3 or DEK provided a hypothesis of long-term sedentary pathogenesis in CRC patients by increasing the protein levels of related biomarkers of CRC.

Some important questions should be considered since the work explores the association between sedentary lifestyle and changes in biomarker expression. Firstly, if the risk factor changes the gene expression, such a trend should be seen in the healthy controls. Our results did not show the significant trend (Fig. 6). There were two reasons for that. (1) There were a few subjects with heavy sedentary in healthy groups compared with those in CRC groups (Table 1). (2) Although there was no statistical difference in sedentary time between $\mathrm{L}$ and $\mathrm{N}, \mathrm{N}$ and $\mathrm{M}$, and $\mathrm{M}$ and $\mathrm{N}$ in healthy subjects, the difference was still found between $\mathrm{L}$ and $\mathrm{H}$. The $P$ values were $0.047,0.039$, 0.045 and 0.025 for Hv1, MXRA5, PIAS3 and DEK respectively. Secondly, based on the data in Table 1, the patients with the most advanced disease also have the highest BMI and more proportion of heavy sedentary. Thus, it is possible that the patients have been with advanced CRC for many years, which contributes to less activity. To reduce the effects, we have surveyed the sedentary time of all patients during the past 10 years before the diagnosis. According to a previous report, most CRC deaths occurred within the first 5 years after diagnosis (Slattery et al. 2011), suggesting that very few advanced cancer patients survive more than 5 years.

In summary, we investigated $228 \mathrm{CRC}$ patients and 80 healthy subjects to reveal the effects of long-term sedentary on the risk of CRC. The length of sedentary was positively related to the levels of biomarkers Hv1, MXRA5, PIAS3 and DEK, which are strongly associated with the risk of CRC. Thus, Hv1, MXRA5, PIAS3 and DEK can be combined targets for curing CRC. Certainly, there are some limitations for the present study: absence of follow up of patients to determine if obesity levels correlate to CRC severity or outcome. Further work need to be done to address such problems in future.

\section{Conflict of Interest}

The authors declare no conflict of interest.

\section{References}

Acott, A.A., Theus, S.A., Marchant-Miros, K.E. \& Mancino, A.T. (2008) Association of tobacco and alcohol use with earlier development of colorectal cancer: should we modify screening guidelines? Am. J. Surg., 196, 915-918.

Bardou, M., Barkun, A.N. \& Martel, M. (2013) Republished: obesity and colorectal cancer. Postgrad. Med. J., 89, 519-533.

Bouwens, M.W., de Ridder, R., Masclee, A.A., Driessen, A., Riedl, R.G., Winkens, B. \& Sanduleanu, S. (2013) Optical diagnosis of colorectal polyps using high-definition i-scan: an educational experience. World J. Gastroenterol., 19, 4334-4343.

Boyle, T., Fritschi, L., Heyworth, J. \& Bull, F. (2011) Long-term sedentary work and the risk of subsite-specific colorectal cancer. Am. J. Epidemiol., 173, 1183-1191.

Crous-Bou, M., Rennert, G., Cuadras, D., Salazar, R., Cordero, D., Saltz Rennert, H., Lejbkowicz, F., Kopelovich, L., Monroe Lipkin, S., Bernard Gruber, S. \& Moreno, V. (2013) Polymorphisms in alcohol metabolism genes ADH1B and ALDH2, alcohol consumption and colorectal cancer. PLoS One, 8, e80158.

Dabir, S., Kluge, A., Aziz, M.A., Houghton, J.A. \& Dowlati, A. (2011) Identification of STAT3-independent regulatory effects for protein inhibitor of activated STAT3 by binding to novel transcription factors. Cancer Biol. Ther., 12, 139-151.

Dabir, S., Kluge, A., McColl, K., Liu, Y., Lam, M., Halmos, B., Wildey, G. \& Dowlati, A. (2014) PIAS3 activates the intrinsic apoptotic pathway in non-small cell lung cancer cells independent of p53 status. Int. J. Cancer, 134, 1045-1054.

Dagvadorj, A., Tan, S.H., Liao, Z., Xie, J., Nurmi, M., Alanen, K., Rui, H., Mirtti, T. \& Nevalainen, M.T. (2010) N-terminal truncation of Stat $5 \mathrm{a} / \mathrm{b}$ circumvents PIAS3-mediated transcriptional inhibition of Stat5 in prostate cancer cells. Int. J. Biochem. Cell Biol., 42, 2037-2046.

Derry, M.M., Raina, K., Agarwal, C. \& Agarwal, R. (2013) Identifying molecular targets of lifestyle modifications in colon cancer prevention. Front. Oncol., 3, 119.

Dunnebier, T., Bermejo, J.L., Haas, S., Fischer, H.P., Pierl, C.B., Justenhoven, C., Brauch, H., Baisch, C., Gilbert, M., Harth, V., Spickenheuer, A., Rabstein, S., Pesch, B., Bruning, T., Ko, Y.D. \& Hamann, U. (2010) Polymorphisms in the UBC9 and PIAS3 genes of the SUMO-conjugating system and breast cancer risk. Breast Cancer Res. Treat., 121, 185-194.

Ferlay, J., Shin, H.R., Bray, F., Forman, D., Mathers, C. \& Parkin, D.M. (2010) Estimates of worldwide burden of cancer in 2008: GLOBOCAN 2008. Int. J. Cancer, 127, 2893-2917.

Gingras, D. \& Beliveau, R. (2011) Colorectal cancer prevention through dietary and lifestyle modifications. Cancer Microenviron., 4, 133-139.

Goh, S. (2013) Systematic review and meta-analysis of the evidence for flexible sigmoidoscopy as a screening method for the prevention of colorectal cancer. Br. J. Surg., 100, 1540.

Guffey, C.R., Fan, D., Singh, U.P. \& Murphy, E.A. (2013) Linking obesity to colorectal cancer: recent insights into plausible biological mechanisms. Curr. Opin. Clin. Nutr. Metab. Care, 16, 595-600.

Heini, A.F. \& Weinsier, R.L. (1997) Divergent trends in obesity and fat intake patterns: the American paradox. Am. J. Med., 102, 259-264.

Howard, R.A., Freedman, D.M., Park, Y., Hollenbeck, A., 
Schatzkin, A. \& Leitzmann, M.F. (2008) Physical activity, sedentary behavior, and the risk of colon and rectal cancer in the NIH-AARP Diet and Health Study. Cancer Causes Control, 19, 939-953.

Hua, Y., Hu, H. \& Peng, X. (2009) Progress in studies on the DEK protein and its involvement in cellular apoptosis. Sci. China, C, Life Sci., 52, 637-642.

Ishii, K., Shibata, A. \& Oka, K. (2013) Identifying environmental, social, and psychological correlates of meeting the recommended physical activity levels for colon cancer prevention among Japanese adults. J. Sci. Med. Sport, 16, 520-525.

Kyro, C., Skeie, G., Loft, S., Overvad, K., Christensen, J., Tjonneland, A. \& Olsen, A. (2014) Adherence to a healthy Nordic food index is associated with a lower incidence of colorectal cancer in women: the Diet, Cancer and Health cohort study ERRATUM. Br. J. Nutr., 111, 758-759.

Li, H., Gao, H., Bijukchhe, S.M., Wang, Y. \& Li, T. (2013) PIAS3 may represent a potential biomarker for diagnosis and therapeutic of human colorectal cancer. Med. Hypotheses, 81, 1151-1154.

Li, Y., Xu, R. \& Dong, J. (2012) Energy expenditure, energy intake and nutritional indices in chinese peritoneal dialysis patients. Kidney Res. Clin. Pract., 31, A51.

Lin, L., Piao, J., Gao, W., Piao, Y., Jin, G., Ma, Y., Li, J. \& Lin, Z. (2013) DEK over expression as an independent biomarker for poor prognosis in colorectal cancer. BMC Cancer, 13, 366-374.

Liu, L.M., Yan, M.G., Yang, D.H., Sun, W.W. \& Zhang, J.X. (2011) PIAS3 expression in human gastric carcinoma and its adjacent non-tumor tissues. Clin. Res. Hepatol. Gastroenterol., 35, 393-398.

Madka, V. \& Rao, C.V. (2013) Anti-inflammatory phytochemicals for chemoprevention of colon cancer. Curr. Cancer Drug Targets, 13, 542-557.

Martínez, M.E., Giovannucci, E., Spiegelman, D., Hunter, D.J., Willett, W.C. \& Colditz, G.A. (1997) Leisure-time physical activity, body size, and colon cancer in women. J. Natl. Cancer Inst., 89, 948-955.

Matthews, C.E., George, S.M., Moore, S.C., Bowles, H.R., Blair, A., Park, Y., Troiano, R.P., Hollenbeck, A. \& Schatzkin, A. (2012) Amount of time spent in sedentary behaviors and cause-specific mortality in US adults. Am. J. Clin. Nutr., 95, 437-445.

Meyerhardt, J.A., Giovannucci, E.L., Holmes, M.D., Chan, A.T., Chan, J.A., Colditz, G.A. \& Fuchs, C.S. (2006) Physical activity and survival after colorectal cancer diagnosis. J. Clin. Oncol., 24, 3527-3534.

Ó Céilleachair, A.J., Hanly, P., Skally, M., O’Neill, C., Fitzpatrick, P., Kapur, K., Staines, A. \& Sharp, L. (2013) Cost comparisons and methodological heterogeneity in cost-of-illness studies: the example of colorectal cancer. Med. Care, 51, 339-350.

Ogata, Y., Osaki, T., Naka, T., Iwahori, K., Furukawa, M., Nagatomo, I., Kijima, T., Kumagai, T., Yoshida, M. \& Tachibana, I. (2006) Overexpression of PIAS3 suppresses cell growth and restores the drug sensitivity of human lung cancer cells in association with PI3-K/Akt inactivation. Neoplasia, $\mathbf{8}$, 817-825.

Peng, X.E., Jiang, Y.Y., Shi, X.S. \& Hu, Z.J. (2013) NQO1 $609 \mathrm{C}>\mathrm{T}$ polymorphism interaction with tobacco smoking and alcohol drinking increases colorectal cancer risk in a Chinese population. Gene, 521, 105-110.

Privette Vinnedge, L.M., Kappes, F., Nassar, N. \& Wells, S.I. (2013) Stacking the DEK: from chromatin topology to cancer stem cells. Cell Cycle, 12, 51-66.
Ramsey, I.S., Mokrab, Y., Carvacho, I., Sands, Z.A., Sansom, M.S. \& Clapham, D.E. (2010) An aqueous $\mathrm{H}+$ permeation pathway in the voltage-gated proton channel Hv1. Nat. Struct. Mol. Biol., 17, 869-875.

Ramsey, I.S., Ruchti, E., Kaczmarek, J.S. \& Clapham, D.E. (2009) Hv1 proton channels are required for high-level NADPH oxidase-dependent superoxide production during the phagocyte respiratory burst. Proc. Natl. Acad. Sci. USA, 106, $7642-$ 7647

Ritvo, P., Myers, R.E., Paszat, L., Serenity, M., Perez, D.F. \& Rabeneck, L. (2013) Gender differences in attitudes impeding colorectal cancer screening. BMC Public Health, 13, 500-513.

Saito, Y., Emura, F., Matsuda, T., Uraoka, T., Nakajima, T., Ikematsu, H., Gotoda, T., Saito, D. \& Fujii, T. (2005) A new sinker-assisted endoscopic submucosal dissection for colorectal cancer. Gastrointest. Endosc., 62, 297-301.

Slattery, M.L., Lundgreen, A., Herrick, J.S., Wolff, R.K. \& Caan, B.J. (2011) Genetic variation in the transforming growth factor- $\beta$ signaling pathway and survival after diagnosis with colon and rectal cancer. Cancer, 117, 4175-4183.

Sobin, L.H., Gospodarowicz, M.K. \& Wittekind, C. (2011) TNM classification of malignant tumours, Blackwell Publishing, New York, NY.

Suchanek, S., Majek, O., Vojtechova, G., Minarikova, P., Rotnaglova, B., Seifert, B., Minarik, M., Kozeny, P., Dusek, L. \& Zavoral, M. (2014) Colorectal cancer prevention in the Czech Republic: time trends in performance indicators and current situation after 10 years of screening. Eur. J. Cancer Prev., 23, 18-26.

Tan, S.C., Suzairi, M.S., Aizat, A.A., Aminudin, M.M., Nurfatimah, M.S., Bhavaraju, V.M., Biswal, B.M. \& Ankathil, R. (2013) Gender-specific association of NFKBIA promoter polymorphisms with the risk of sporadic colorectal cancer. Med. Oncol., 30, 693-696.

Temraz, S., Mukherji, D. \& Shamseddine, A. (2013) Potential targets for colorectal cancer prevention. Int. J. Mol. Sci., 14, 17279-17303.

Vermorken, A.J., Zhu, J. \& Andres, E. (2014) Obesity and colorectal cancer risk: the role of oxidative stress. Gut, $\mathbf{6 3}$, 529-530.

Wang, G.H., Yao, L., Xu, H.W., Tang, W.T., Fu, J.H., Hu, X.F., Cui, L. \& Xu, X.M. (2013a) Identification of MXRA5 as a novel biomarker in colorectal cancer. Oncol. Lett., 5, 544-548.

Wang, Y., Wu, X., Li, Q., Zhang, S. \& Li, S.J. (2013b) Human voltage-gated proton channel hv1: a new potential biomarker for diagnosis and prognosis of colorectal cancer. PLoS One, $\mathbf{8}$, e70550.

Wise-Draper, T.M., Allen, H.V., Jones, E.E., Habash, K.B., Matsuo, H. \& Wells, S.I. (2006) Apoptosis inhibition by the human DEK oncoprotein involves interference with p53 functions. Mol. Cell. Biol., 26, 7506-7519.

Xiong, D., Li, G., Li, K., Xu, Q., Pan, Z., Ding, F., Vedell, P., Liu, P., Cui, P. \& Hua, X. (2012) Exome sequencing identifies MXRA5 as a novel cancer gene frequently mutated in nonsmall cell lung carcinoma from Chinese patients. Carcinogenesis, 33, 1797-1805.

Yokoyama, J., Ajioka, Y., Watanabe, H. \& Asakura, H. (2002) Lymph node metastasis and micrometastasis of submucosal invasive colorectal carcinoma: an indicator of the curative potential of endoscopic treatment. Acta Med. Biol., 50, 1-8.

Zhao, J., Halfyard, B., Roebothan, B., West, R., Buehler, S., Sun, Z., Squires, J., McLaughlin, J.R., Parfrey, P.S. \& Wang, P.P. (2010) Tobacco smoking and colorectal cancer: a populationbased case-control study in Newfoundland and Labrador. Can. J. Public Health, 101, 281-289. 